respectively. $\mathrm{R}^{2}$ varied across states, though positive, from 0.29 in Akwa Ibom to 0.95 in Kebbi states. Standard deviation of residuals in the regression model ranged from -3.89 to 3.33 in Borno and Gombe states respectively, while Sokoto and Bauchi had 0.006 and 0.024 respectively, thus having the best accuracy in predictions across all states in the country. Both correlation and GWR were at $\mathrm{p}<0.05$.

Conclusion The results obtained support literature, confirming the inverse relationship between ORST prevalence, improved drinking water access and improved sanitation to diarrhea prevalence. It also supports the already confirmed positive relationship between poor nutrition of children and susceptibility to diarrhoea. The study however expanded knowledge by incorporating geocomputation to predict diarrhoea prevalence.

\section{PO 8438 KNOWLEDGE, ATTITUDE AND PERCEPTIONS ON ADVERSE DRUG EVENTS REPORTING AMONG PATIENTS AND HEALTHCARE PROVIDERS IN RURAL UGANDA}

${ }^{2}$ Helen Ndagije, ${ }^{2}$ Victoria Nambasa, ${ }^{1}$ Dan Kajungu. 'Makerere University Centre for Health and Population Research-lganga Mayuge HDSS, Kampala, Uganda; ${ }^{2}$ National Drug Authority, Kampala, Uganda

\subsection{6/bmjgh-2019-EDC.96}

Background Drug regulatory authorities promote patient safety by, among other ways, monitoring adverse drug events (ADEs). Reporting of ADEs in Uganda is below the average for a well-performing system. Enhancing patients' understanding of and involving them in reporting ADEs improves drug safety and treatment outcome monitoring. The objective of this study was to describe the knowledge, attitude, and practice of patients and healthcare workers regarding ADEs and ADEs reporting.

Methods A cross-sectional survey was carried out among 1034 respondents from randomly selected households and 327 health workers at health facilities in the Iganga Mayuge Health and Demographic Surveillance Site (IMHDSS). The IMHDSS, located in Uganda, covers 90,000 people living in 17,000 households.

Results Over half of respondents (59\%) sought treatment from private drug shops, $37 \%$ from either clinic, health center or hospital, while $4 \%$ sought treatment from herbalists, friends or relatives. Over half (56\%) were aware of ADEs, 57\% expressed willingness to report an ADE while 43\% did not know what to do when it occurs. Almost half (46\%) could not differentiate between an $\mathrm{ADE}$ and the symptoms, and for those who could, the majority (76\%) were willing to report it. Only 34\% had ever reported an ADE when it occurred to them. Of those who reported, $43 \%$ had their drugs changed, $31 \%$ were only counseled while $11.5 \%$ continued taking the same medication. Among healthcare workers, 95\% knew about ADEs, but only $35 \%$ had ever reported. Reasons for not reporting were: fear of being victimised or sued (35\%); lack of adequate knowledge about ADE (26\%); 20\% thought it would disappear shortly; and $14 \%$ did not find it necessary to report.

Conclusion Patients seek their treatment from private providers. Patients want to report ADEs, but they do not have adequate knowledge. Healthcare workers' reasons for not reporting are subjective. Dedicated pharmacovigilance-related interventions at community level would improve community members' knowledge and hence ADE reporting rate.

\section{PO 8439 EFFECT OF PLASMODIUM FALCIPARUM EXPOSURE ON HUMAN URINARY METABOLOMICS PROFILING}

${ }^{1}$ Madeleine E Betouke Ongwe*, ${ }^{2}$ Aswin Verhoeven, ${ }^{1}$ Akim Ayola Adegnika, ${ }^{2}$ Maria Yazdanbakhsh, ${ }^{2}$ Oleg Mayboroda. ${ }^{1}$ Centre de recherche médicale de Lambarene, Libreville, Gabon; 'Leiden University Medical Center, Netherlands

\subsection{6/bmjgh-2019-EDC.97}

Background Immunity against malaria infection is being studied extensively but the underlying mechanisms of protection remain not fully understood. Metabolomics is a postgenomic technology enabling a minimally invasive monitoring of the physiological responses to external and internal stimuli. Here, we present a longitudinal study of the urinary metabolic profiles of healthy individuals before and after intravenous administration of $P$. falciparum sporozoïtes, aiming at deciphering the metabolic changes observed during malaria infection.

Methods Twenty (20) healthy Gabonese and 5 Europeans were voluntary challenged by live $P$. falciparum sporozoïtes (3200 PfSPZ) and followed up until they developed symptoms and became thick blood smear-positive. Urine samples were collected before and after challenge at several time points until treatment. Samples were analysed in an untargeted approach using state-of-the-art analytical platforms, namely hydrophilic interaction chromatography-mass spectrometry (HILIC-MS) and nuclear magnetic resonance (NMR) spectroscopy. A combination of the multivariate and univariate data analysis approaches was used for dissecting the metabolic effects of a host response to the infection.

Results Unlike the Europeans participants, a part of the Gabonese volunteers did not become parasitaemic. Unsupervised data analysis shows sample discrimination between Europeans and Gabonese at baseline, before and after challenge and between Gabonese who controlled their parasitaemia and those who did not.

Conclusion This metabolomics study highlighted the differences in the urinary metabolite profiles during $P$. falciparum infection. These differences observed between parasitaemic and non-parasitaemic Gabonese after challenge with P. falciparum, may suggest an underlying metabolic mechanism of protection against malaria infection which we will investigate in detail.

\section{PO 8441 EXPERIMENTAL COMPARISON OF SENSITIVITY OF LAMP AND REAL-TIME PCR}

${ }^{1}$ Jacques Kaboré*, ${ }^{2}$ Hamidou Ilboudo, ${ }^{1}$ Charlie FA Compaoré, ${ }^{2} \mathrm{O}$ umou Camara, ${ }^{1}$ Mohamed Bamba, 'Hassane Sakandé, ${ }^{2}$ Mamadou Camara, ${ }^{2}$ Bruno Bucheton, ${ }^{3}$ Dramane Kaba, ${ }^{3}$ Vincent Jamonneau, ${ }^{4}$ Stijn Deborggraeve, ${ }^{5}$ Veerle Lejon. ${ }^{1}$ Centre International de Recherche-Développement sur I'Elevage en zone Subhumide, BoboDioulasso, Burkina Faso; ${ }^{2}$ Programme National de Lutte contre la THA, Guinea; ${ }^{3}$ Institut Pierre Richet, Bouajem, Côte d'Ivoire; ${ }^{4}$ Institut de Médecine Tropicale, Antwerp, Belgium; ${ }^{5}$ Institut de Recherche pour le Developpement, Marseille, France

\subsection{6/bmjgh-2019-EDC.98}

Background Human African trypanosomiasis, or sleeping sickness, remains a serious problem in tropical Africa. Timely diagnosis of this disease requires systematic population screening, particularly for Trypanosoma brucei gambiense, which has a long asymptomatic period.

The lack of sensitivity and specificity of conventional diagnostic tests has led in recent years to the use of molecular tools. Amplification of parasite-specific DNA sequences 